\title{
El consumo moderado de alcohol reduciría la incidencia de eventos cardiovasculares
}

\author{
Moderate alcohol consumption may reduce the incidence of cardiovascular events
}

Bell S. BMJ 2017;356:j909.

\section{Objetivos}

Investigar la asociación entre cinco categorías de consumo de alcohol y la presentación inicial de enfermedad cardiovascular (ECV).

\section{Diseño}

Estudio de cohortes de base poblacional.

\section{Lugar}

Bases de datos electrónicas de centros de Atención Primaria, Hospitales y la Oficina Nacional de Estadísticas de Inglaterra.

\section{Pacientes}

1.937.360 adultos (51\% mujeres), mayores de 30 años sin ECV al inicio del estudio. El seguimiento promedio fue de seis años.

\section{Evaluación de los factores de riesgo}

Se definieron cinco categorías de bebedores: no bebedores, ex bebedores, bebedores ocasionales, bebedores moderados y bebedores intensos. Las covariables consideradas en los análisis multivariables incluyeron edad, sexo, nivel socioeconómico, tabaquismo, diabetes, presión arterial sistólica, índice de masa corporal, lipoproteínas de alta densidad, uso de antihipertensivos o estatinas, y si el paciente había recibido recomendaciones alimentarias.

\section{Medición de resultados principales}

Se registró el primer diagnóstico de angina crónica estable, angina inestable, infarto de miocardio, muerte súbita por enfermedad coronaria, insuficiencia cardíaca, paro cardiaco de causa coronaria, accidente isquémico transitorio, accidente cerebrovascular isquémico, hemorragia intracerebral, hemorragia subaracnoidea, enfermedad arterial periférica y aneurisma de aorta abdominal. Adicionalmente, se estimaron asociaciones con mortalidad de causa no-cardiovascular.

\section{Resultados principales}

El consumo moderado de alcohol mostró un efecto protector para enfermedad coronaria, infarto agudo de miocardio, enfermedad arterial periférica, insuficiencia cardiaca y aneurisma de aorta abdominal. No se demostró un efecto protector del consumo moderado de alcohol para accidente isquémico transitorio, accidente cerebrovascular isquémico y hemorragia intracerebral. Para hemorragia subaracnoidea el riesgo en las cinco categorías fue similar. La interpretación de los resultados no cambió sustancialmente cuando se ajustó por las covariables antes mencionadas. Los resultados se muestran en la Tabla 1.

Tabla 1. Eventos cardiovasculares y mortalidad por todas las causas de acuerdo a categorías de consumo de alcohol.

\begin{tabular}{|c|c|c|c|}
\hline Categorias de bebedor & $\begin{array}{l}\text { Enfermedad cardiovascular } \\
\text { fatal y no-fatal }\end{array}$ & $\begin{array}{l}\text { Enfermedad } \\
\text { cardiovascular fatal }\end{array}$ & $\begin{array}{c}\text { Mortalidad por todas las } \\
\text { causas }\end{array}$ \\
\hline No bebedor & $1,23(1,19$ a 1,27$)$ & $1,32(1,27$ a 1,38$)$ & $1,24(1,20$ a 1,28$)$ \\
\hline Ex bebedor & $1,29(1,22$ a 1,35$)$ & $1,44(1,28$ a 1,62$)$ & $1,38(1,30$ a 1,47$)$ \\
\hline Bebedor ocasional & $1,10(1,07$ a 1,13$)$ & $1,09(1,03$ a 1,16$)$ & $1,05(1,03$ a 1,07$)$ \\
\hline Bebedor moderado & 1,00 (referencia) & 1,00 (referencia) & 1,00 (referencia) \\
\hline Bebedor intenso & $1,14(1,10$ a 1,19$)$ & $1,20(1,13$ a 1,27$)$ & $1,34(1,31$ a 1,98$)$ \\
\hline
\end{tabular}

\section{Conclusiones}

El consumo moderado de alcohol está asociado con un menor incidencia de varias, pero no todas, las formas de presentación de la ECV.

Fuente de financiamiento/conflicto de interés de los autores: National
Institute for Health Research, Wellcome Trust, Medical Research Council, Arthritis Research UK, British Heart Foundation, Cancer Research UK, Chief Scientist Office, Economic and Social Research Council, Engineering and Physical Sciences Research Council, National Institute for Social Care and Health Research, European Research Council.

\section{Comentario}

Este estudio es el primero que discrimina entre no bebedores, ex bebedores y bebedores moderados respecto de la incidencia de ECV. Aunque se ha planteado que los efectos protectores del consumo moderado de alcohol han sido sobreestimados ${ }^{1}$, este estudio confirma la asociación en curva "J" entre consumo de alcohol y algunos eventos cardiovasculares (mayor riesgo para no bebedores y bebedores intensos). Sin embargo, sus conclusiones no deben interpretarse como fundamento para recomendar la ingesta moderada de alcohol. Desde que el Efecto Roseto fue descripto en $1961^{2}$, la enfermedad cardiovascular ha sido una gran excusa a través de la cual adentrarse en la epidemiología sociocultural. Si bien este no es un ensayo clínico, sería interesante postular que cierto "efecto del adherente sano" ${ }^{3}$ pueda explicar que el potencial efecto protector que se observa en los bebedores moderados quizás no se deba a las propiedades químicas del alcohol propiamente dicho, sino a que éstos bebedores también sean "moderados" en otras actividades de su vida, y tengan menos accidentes de tránsito, menos divorcios, menos problemas financieros, más amigos, sean más propensos a vacunarse, y todo lo que hace que una persona sea más saludable. Es lo que Michel Foucault llamaba "Cuidado de Sí"4.

\section{Conclusiones del comentador}

Si bien este estudio confirma la asociación en curva "J" entre el consumo de alcohol y la ECV, esta asociación no debe interpretarse como fundamento para recomendar la ingesta moderada de alcohol.

Agustín González Calbano [ Departamento de Ciencias de la Salud. Universidad Nacional del Sur. agustin.gonzalez@uns.edu.ar] González Calbano A. El consumo moderado de alcohol reduciría la incidencia de eventos cardiovasculares. Evid Act Pract Ambul 2018;21(2):59. Comentado de: Bell S y col. Bariatric Surgery versus Intensive Medical Therapy in Obese Patients with Diabetes. Association between clinically recorded alcohol consumption and initial presentation of 12 cardiovascular diseases: population based cohort study using linked health records. BMJ. 2017 Mar 22;356:j909 PMID: 28331015. 